\title{
The More Reliable Path for Post Great Earthquake Traffic Management
}

\author{
Tien-Pen Hsu, \\ Institute of Civil Engineering \\ National Taiwan University \\ Taipei Roosevelt Road Sec. 4 No. 1 \\ Email: hsutp@ntu.edu.tw
}

\author{
Wen-Shen Jung, \\ Institute of Civil Engineering \\ National Taiwan University \\ Taipei Roosevelt Road Sec.4 No.1 \\ Email: r8521524@dove.cc.ntu.edu.tw
}

\begin{abstract}
In this paper, a "Double Label Algorithm" method is created to seek a more reliable path and a minimal travel time path in a stochastic time dependent network. The idea of this paper comes from the work to dispatch the rescue vehicle to disaster area post earthquake, since the network will become unreliable due to damage by earthquake. The concept of the method is developed based on the analysis of travel time of a stochastic and time-varying network. The paper first identifies a set of relationships between the mean and variance of the travel time dynamic and stochastic in network. A shortest path is generated as more reliable path under consideration of the travel time variance and time-varying features of the arrival time at the destination. Comparing the travel time of the shortest travel time path with the travel time of the more reliable path, a reliability index can be obtained and taken as link weight to generate routing decision.
\end{abstract}

Keywords: Reliability, shortest path, stochastic network, traffic management, earthquake.

\section{Introduction}

The Chi-Chi great earthquake occurred on 21st September 1999 in the suburban areas in the Central Taiwan. All rescue action should use the transportation system from outside to reach the disaster areas. The part of transportation system was blocked by the collapsed buildings, fault rupture and falling bridges. It causes the decrease of the capacity of transportation system. The uncertainty of the network performance rises significantly. For dispatching the rescue vehicle to disaster area become more complicated. Nevertheless, the increased travel demand and concentrated traffic volume to specific destination make some popular streets overloading, meanwhile the transportation system capacity decreased causes the serious traffic congestion during the rescue period. The network performance in terms of travel time becomes a stochastic issue due to the uncertainty of street service situation and the stochastic varying travel demand. Therefore, to create a model to generate a path of more reliability is necessary for enhancing the rescue work performance during the post great earthquake period.
The transportation network post earthquake can be seen as a stochastic and time-varying network. In a stochastic, time-varying transportation network the travel time on the network is represented as random variables. The probability distribution functions with vary with time arrived at the origin of the link. In recent years, there are several papers addressed the problem $[2,3,4,5,6]$. However, the researches considered only the shortest path of time-dependent problems, didn't concern about the probability. This study considers stochastic, time-varying transportation network, where the travel time on the network should be represented as random variables and aim at seek a path to ensure the rescue vehicle can reach the destination under a certainty. The means, the reliability of the path will be taken into account. The path with high reliability is not necessary to be a minimal travel time shortest path. It implicates using this path with high reliability the arrival time before a certain time point is ensured, i.e. more reliable. The probability distribution functions of each link vary with time, i.e. time dependent. Therefore, in this paper identifies a set of relationships between the mean and variance of the travel time that is dynamic and stochastic in network. Upon these relationships, compute the reliability index of arriving destination in time, and use the index as link weight to generate routing decisions. Lastly, analysis the trade-off relation between travel time and reliability, provide different routing paths for road users or the rescue work manager to make decisions. This paper introduces reliability evaluation method, discussing how to determine the most reliable path arriving destination in time in the dynamic and time dependent transportation networks.

\section{Mathematical formulas}

\subsection{Travel time formula of Fu and Rillett}

The paper defined links travel time as continuous random variable and its probability distribution functions depend on the arriving time following the concept from Fu and Rilett's research result [2]. Assuming $G=(V$, $A, J, T$ ) is a directed graph where $V$ is the set of nodes, $|V|=v$, and $A$ is the set of $\operatorname{arcs},|A|=m$. Link travel time is 
a continuous random variable. The mean of stochastic process $\left\{X_{a}(t), t \in T\right\}$ corresponding to its first-order PDF is represented by $\mu_{X_{a}}(t)$ and is defined as follows.

$\mu_{X_{a}}(t)=E\left[X_{a}(t)\right]=\int_{0}^{+\infty} x_{a} f_{X_{a}}\left(x_{a}, t\right) d x_{a}$

As the same, the variance of travel time $V X_{a}(t)$ could be represented as follows :

$$
v_{X}(t)=E\left[\left(X_{a}(t)-\mu_{X}(t)\right)^{2}\right]=\int_{0}^{+\infty}\left(x_{a}-\mu_{X}(t)\right)^{2} f_{X}\left(x_{a}, t\right) d x_{a}
$$

A two-link network is shown in Figure 1.

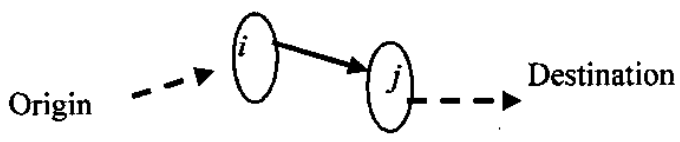

Figure1. A two-link dynamic network.

Where:

Yi : the arrival time at node $i$

E(Yi) : the expected time to node $\mathrm{i}$

$\operatorname{Var}(Y i):$ the variance to node $i$

The approximated model of arrival time can be denoted as

$E\left[Y_{j}\right]=E\left[Y_{i}\right]+E\left[Z_{a}\right]=E\left[Y_{i}\right]+E\left[E\left[Z_{a} \mid Y_{i}\right]\right]$

$\cong E\left[Y_{i}\right]+\mu_{X_{0}}\left(E\left[Y_{i}\right]\right)+\frac{1}{2} \mu_{X_{a}}^{\prime \prime}\left(E\left[Y_{i}\right]\right) \cdot \operatorname{Var}\left[Y_{i}\right]$

The arrival time to node $\mathrm{j}$ is $\mathrm{Yj}$, the approximated model of arrival time variance $\operatorname{Var}\left[\mathrm{Y}_{\mathrm{j}}\right]$ can be denoted as

$$
\begin{aligned}
& \operatorname{Var}\left[Y_{j}\right] \\
& \cong\left\{\left(1+\mu_{\mathrm{X}_{\mathrm{a}}^{\prime}}^{\prime}\left(\mathrm{E}\left[\mathrm{Y}_{\mathrm{i}}\right]\right)\right)^{2}+\frac{1}{2} \mathrm{v}_{\mathrm{X}_{\mathrm{a}}^{\prime \prime}}\left(\mathrm{E}\left[\mathrm{Y}_{\mathrm{i}}\right]\right)\right] \\
& \left.-\frac{1}{4} \mu_{\mathrm{X}_{\mathrm{a}}^{\prime \prime 2}}\left(\mathrm{E}\left[\mathrm{Y}_{\mathrm{i}}\right]\right) \cdot \operatorname{Var}\left[\mathrm{Y}_{\mathrm{i}}\right]\right\} \cdot \operatorname{Var}\left[\mathrm{Y}_{\mathrm{i}}\right]+v_{\mathrm{X}_{\mathrm{a}}}\left(\mathrm{E}\left[\mathrm{Y}_{\mathrm{i}}\right]\right) \\
& +\left(1+\mu_{X_{a}}^{\prime}\left(E\left[Y_{i}\right]\right)\right) \cdot \mu_{X_{a}}^{\prime \prime}\left(E\left[Y_{i}\right]\right) \cdot\left(\beta_{1} \operatorname{Var}^{3}\left[Y_{i}\right]\right)^{\frac{1}{3}} \\
& +\frac{1}{4} \mu_{X_{a}}^{\prime 2}\left(E\left[Y_{i}\right]\right) \cdot \beta_{2} \operatorname{Var}^{2}\left[Y_{i}\right] .
\end{aligned}
$$

\subsection{Travel time multi-distribution formula}

For simplifying the algorithm and to match the traffic situation of different periods, such as at peak hour or at non peak hour, a multi-distribution concept is adopted in this paper. Fu's model assumes travel time is continuous, differentiable random variable. According to the traffic flow theory, the traffic flow from noncongestion to congestion would jump occasionally. Therefore, this paper divided travel period of a link into several timer intervals. In each time interval, travel time of the link is continuous and differentiable random variable, and its PDF is to be assumed as normal distribution. The links travel time is defined as continuous random variable and its probability distribution functions depend on the arriving time.

Assumed $\mathrm{G}=(\mathrm{V}, \mathrm{A}, \mathrm{J}, \mathrm{T})$ be a directed graph where $V$ is the set of nodes, $|V|=v$, and $A$ is the set of arcs, $|A|=m$. Link travel time is continuous random variable. $T$ is the time range examined although for generality it will be assumed that $T=R+=(0, \infty)$. To separate $T$ to several time interval, $t_{0} \leq t \leq t_{0}+k t$, $k=1 \cdot 2 \cdot \ldots \cdot n,\{t \in T\}$. In each time interval $k, X a(t)$ is regarded as a continuous random variable $\{\mathrm{Xa}(\mathrm{t})$, $t \in T$ ). The PDF $f_{X_{a}}\left(x_{a}, t\right)$ is normal distribution $N\left(\mu_{X_{a}}(t), V X_{a}(t)\right)$.

The function of expected travel time $\mu_{X_{a}}(t)$ is denoted as

$\mu_{X_{a}}(t)=E\left[X_{a}(t)\right]=\sum_{i=0}^{n} \int_{i_{i}}^{t_{i}+\Delta t} x_{a} f_{X_{a}}\left(x_{a}, t_{i}\right) d x_{a}$

The variance of travel time $V \mathrm{X}_{\mathrm{a}}(\mathrm{t})$ is denoted as :

$v_{X_{i}}(t)=\mathrm{E}\left[\left(X_{a}(t)-\mu_{X_{a}}(t)\right)^{2}\right]=\sum_{i=0}^{n} \int_{t_{i}}^{t_{i}+\Delta t}\left(x_{a}-\mu_{X_{a}}\left(t_{i}\right)\right)^{2} f_{X_{a}}\left(x_{a}, t_{i}\right) d x_{a}$

The travel time from node $\mathrm{i}$ to node $\mathrm{j}$ is $\mathrm{Yj}=\mathrm{Yj}+$ $\mathrm{Za}$, The expected value of $\mathrm{Yj}$ can be represented as follows

$$
E\left[Y_{j}\right]=\sum_{i=1}^{n}\left[\int_{t_{i}}^{t_{i}+\Delta t} f_{x_{i}}\left(x_{i}, t_{i}\right) d_{x_{i}} \times\left(\frac{\mu_{x_{i}}\left(t_{i}\right)+\mu_{x_{j}}\left(t_{i}\right)}{2}\right)\right]
$$




$$
\operatorname{Var}\left(Y_{j}\right)=\sum_{i=1}^{n}\left\{\left[\int_{t_{i}}^{t_{i}+\Delta t} f_{x_{i}}\left(x_{j}, t_{i}\right) d_{x_{i}}\right]\left[\frac{\sigma_{x_{i}}^{2}+\dot{\sigma}_{x_{i}}^{2}}{4}\right]\right\}
$$

\subsection{Reliability index}

The discussion above only considers estimated link travel time. The travel time in each interval is represented by its mean and variance. However, the real and estimated travel time will probably be not consistent. The road users will also concern if he can arrive destination in time or not. This problem implicates reliability. Therefore, in terms of the reliability, this paper discusses a network reliability index.

According to Ang and Tang's model [1], the path travel time $Y$ is summed up by each interval $Y=\sum_{i=1}^{n} y_{i}$. To simplify the problem, if the expectation of travel time is $\mathrm{X}_{0}$, and if each random variable of $\mathrm{yl} \cdot \mathrm{y} 2 \cdot \mathrm{y} 3 \cdots \cdots$ yn has correlation, the reliability index $\beta$ is as follows:

$$
\beta=\mathrm{d}=\frac{X_{0}-\sum_{i=1}^{n} y_{i}}{\sqrt{\sum_{i=1}^{n} \sum_{i=1}^{n} \rho_{i j} \sigma_{y_{i}} \sigma_{y_{j}}}}
$$

The $\beta$ is a random variable of standard normal distribution. The probability $P=Z(\beta)$ is then defined as the probability of the vehicle arriving the end destination before expectation travel time $\mathrm{X}_{0}$

Using $\beta$ as routing decision factor, the more $\beta$ is, the higher the reliability is. As the result, the path might not be the shortest one. Given the expected travel time, the probability of arriving in time is highest.

\section{Double label algorithm}

Because of the time varying features, the standard algorithm of shortest path can't have the optimal solution, so this paper apply Yen's K shortest path algorithm to find the shortest average travel time and the more reliable path.

The $\mathrm{K}$ shortest path algorithm could include the shortest and the more reliable path, but hard to code. The operation time of seeking the path increases with the scale of the network. Therefore, in this paper constructs a new algorithm, called "Double Label Algorithm". The algorithm could solve the shortest path in stochastic networks, and meanwhile generate the most reliable path linked with the shortest path at the same time. This method can operate with less time than using $\mathrm{K}$ shortest path method. The main structure of the algorithm is to modify label correcting algorithm using two principles at the same time to set labels. The first principle is to select the shortest path conventionally. The other one is to select the upstream path's travel time, defined as cost, if it has the value more than the minimal travel time and less than a given parameter $M$, meanwhile travel time variance less than the variance of the shortest path at this stage. Each node of the networks will have two labels. The steps of the algorithm are described as follows.

Parameter definition:

$\mathrm{Q}:$ Queue, store waiting node

$S:$ the value of labels, $S(1)$ the minimum travel time. $S(2)$ the minimum variance of the travel time.

$\mathrm{U}:$ Array to store the order of path from the start node, $\mathrm{U}(1)$ store the order of shortest paths nodes, $\mathrm{U}(2)$ store the order of the path satisfying the second principle.

Dij : the travel time of the link $i j$

$\sigma_{i j}^{2}:$ the variance of the link $i j$ 's travel time

M : the quotient of the minimum travel time divided by current path travel time

Algorithm steps:

Step 0: Initialization and creation of the queue $Q$, Inserting starting node and labeling the starting node $S_{0}(1)=0$. The other nodes labels $S_{i}(1)=\infty$. Determine the value of $\mathrm{M}$.

Stepl: If $Q$ is not empty, select fist node from $Q$. Set the associated node $i$ as the current node. If $Q$ is empty, go to step4.

Step2: Check whether the downstream node $j$ from node $i$ satisfy the following two criteria or not. If satisfied, update the label of node $j$ and insert node $j$ to Q.

Two criteria are:

(1) If $\left(S_{i}(k)+D_{i j}\right)<S_{j}(1)$, setting $S_{j}(1)=S_{i}(k)+D_{i j} k=1,2$

(2) If $\left(\mathrm{S}_{\mathrm{i}}(\mathrm{k})+\mathrm{D}_{\mathrm{ij}}\right)>\mathrm{S}_{\mathrm{j}}(1),\left(\sigma_{\mathrm{Sj}}{ }^{2}+\sigma_{\mathrm{ij}}{ }^{2}\right)<\sigma_{\mathrm{Si}}{ }^{2}$ and $\left(\mathrm{S}_{\mathrm{i}}(\mathrm{k})+\mathrm{D}_{\mathrm{ij}} / \mathrm{S}_{\mathrm{j}}(1)\right)<\mathrm{M}$, setting $\mathrm{S}_{\mathrm{j}}(1)=\mathrm{S}_{\mathrm{i}}(\mathrm{k})+\mathrm{D}_{\mathrm{ij}}$ 。

Step 3: Return to step2, do iteration.

Step4: If $Q$ is a empty queue, then stop. 
Example: in the network in Figure 2, assume the travel times of links are independent just for illustrating the double label algorithm. In the real network, the travel time will be dependent and using the aforementioned formulas to calculate it.

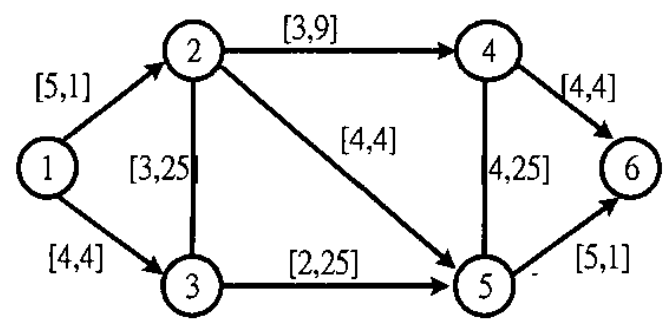

Note: [mean of travel time, variance of travel time]

Figure 2.Example network.

Step0: Inserting starting node and labeling the starting node $S_{0}(1)=0$. The other nodes labels $S_{i}(1)=\infty$. Assume the value of $M=1.5$ for regulating the varying path with a certain limit by shortest path.

Stepl Select fist node 1 from $Q$, Check whether the downstream node 2 and node 3 from node 1 .

$S_{1}(1)+D_{12}=5<S_{2}(2)=\infty, S_{2}(1)=[5,1]$

Upstream node order $U_{2}(1)=\{1\}, Q=\{2\}$

$S_{1}(1)+D_{13}=4<S_{3}(1)=\infty, S_{2}(1)=[4,4]$

Upstream node order $U_{3}(1)=\{1\}, Q=\{2,3\}$

Step2 : Select fist node 2 from $Q$

$S_{2}(1)+D_{24}=8<S_{4}(1)=\infty \cdot S_{4}(1)=[8,10]$

Upstream node order $\mathrm{U}_{4}(1)=\{1,2\}, Q=\{3,4\}$

$S_{2}(1)+D 25=9<S_{5}(1)=\infty, S_{5}(1)=[9,5]$

Upstream node order $U_{5}(1)=\{1,2\}, Q=\{3,4,5\}$

Step3: Select fist node 3 from Q

$\mathrm{S}_{3}(1)+\mathrm{D}_{32}=7>\mathrm{S}_{2}(1)=4$, and variance $29>1$, label unchanged

$\mathrm{S}_{3}(1)+\mathrm{D}_{35}=6<\mathrm{S}_{5}(1)=9, \mathrm{~S}_{5}(1)=[6,29]$

Upstream node order $U_{5}(1)=\{1,3\}, Q=\{4,5\}$

But $S_{5}(1)=[9,5]$, the variance equal to 5 less than $S_{5}(1)=[6,29]$, the variance equal to 29
$9 \div 6=1.5 \leqq M$, according to the second criterion, let $\mathrm{S}_{5}(2)=[9,5]$

Upstream node order $\mathrm{U}_{5}(2)=\{1,2\}, \mathrm{Q}=\{4,5\}$

Step4: Select first node 4 from Q

$\mathrm{S}_{4}(1)+\mathrm{D}_{45}=12>\mathrm{S}_{5}(1)=6$, and the variance equal to $35>29$ label unchanged

$\mathrm{S}_{4}(1)+\mathrm{D}_{46}=12<\mathrm{S}_{1}(1)=\infty, \mathrm{S}_{6}(1)=[12,14]$

Upstream node order $U_{6}(1)=\{1,2,4\}, Q=\{5,6\}$

Step5: Select fist node 5 from Q

$\mathrm{S}_{5}(1)+\mathrm{D}_{56}=11<\mathrm{S}_{6}(1)=12, \mathrm{~S}_{6}(1)=[11,30]$

Upstream node order $U_{6}(1)=\{1,3,5\}, Q=\{6\}$

Meanwhile node 5 has second label, calculate the travel time of downstream of node $5, S_{5}(2)+D 56=14$ $>\mathrm{S}_{6}(1)=11$, the variance equal to $6<30$, and $14 / 11=1.27<1.5$ according to the second criterion. Setting second label of node $6 S_{6}(1)=[14,6]$

Upstream node order $\mathrm{U}_{6}(2)=\{1,2,5\}, Q=\{6\}$

Step6: $Q$ is an empty queue, then stop.

Node 6 has two label $S_{6}(1)=[11,30], S_{6}(2)=[14,6]$ and two node-streams of path are $\mathrm{U}_{6}(1)=\{1,3,5\}$. $U_{6}(2)=\{1,2,5\}$ representing the shortest path take 11 units to travel and the most reliable path take 14 units to travel. The shortest path of travel time is $1-3-5-6$, and the more reliable path is $1-2-5-6$.

\section{Case Study}

\subsection{Using the Fu and Rilett's Formular}

Using the street network in Taipei city center as example the comparison between different shortest path algorithms based on different travel time estimation formulas is conducted. In this case, to simplify the problem, assume that the distributions of each link travel time are identical. Select one road of Taipei as a representative street to get the time dependent travel time function. The distribution of travel time is shown in Figure 3. 


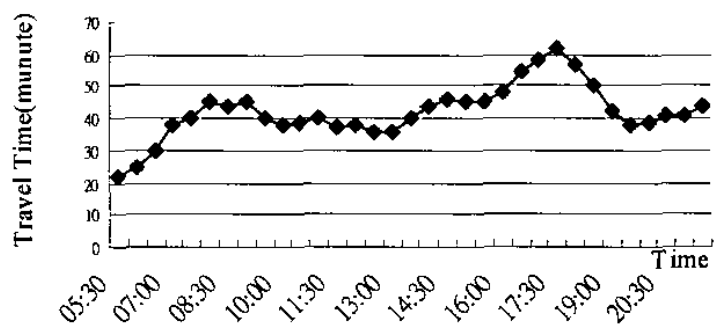

Figure 3. Time dependent travel time, surveyed in Taipei.

Select peak hour time from AM 7:00 to AM 10:00 as example, assume it is a quadratic equation

$\mathrm{Y}=\mathrm{AX}^{2}+\mathrm{BX}+\mathrm{C}$, where $X$ is arrival time of the link, and $Y$ is the travel time of link $i j$. According to the survey results,

$$
\begin{aligned}
& \hat{Y}=-0.00087 X^{2}+0.157143 X+37.5 \\
& R^{2}=0.868
\end{aligned}
$$

Since the distributions of each link are assumed identical, the difference of each link can be identified using the parameter $\mathrm{C}$ in the equation. $\mathrm{C}$ can be seen as the travel time of each link during off-peak time. The travel time regression equation of each link is then as $\hat{Y}_{i}=-0.00087 X^{2}+0.157143 X+C_{i}$

First, using the travel time dependent equation to apply Fu and Rilett's method to calculate the expectation travel time and variance with time-varying

$$
\begin{aligned}
& E\left[Y_{j}\right]=E\left[Y_{i}\right]+E\left[Z_{a}\right]=E\left[Y_{i}\right]+E\left[E\left[Z_{a} \mid Y_{i}\right]\right] \\
& \cong E\left[Y_{i}\right]+\mu_{X_{a}}\left(E\left[Y_{i}\right]\right)+\frac{1}{2} \mu_{X_{a}}^{\prime \prime}\left(E\left[Y_{i}\right]\right) \cdot \operatorname{Var}\left[Y_{i}\right]
\end{aligned}
$$

The second order approximation of arrival time variance is as below:

$$
\begin{aligned}
& \operatorname{Var}\left[\mathrm{Y}_{j}\right] \cong(\mathrm{A}+\mathrm{B}) \operatorname{Var}\left[\mathrm{Y}_{\mathrm{i}}\right]+v_{\mathrm{X}_{\mathrm{a}}}\left(\mathrm{E}\left[\mathrm{Y}_{\mathrm{i}}\right]\right) \\
& \mathrm{A} \cong\left\{1+\mu_{\mathrm{X}_{\mathrm{s}}}^{\prime}\left(\mathrm{E}\left[\mathrm{Y}_{\mathrm{i}}\right]\right)\right\}^{2} \\
& \mathrm{~B}=\frac{1}{2}\left\{v_{\mathrm{X}_{\mathrm{a}}}^{\prime \prime}\left(\mathrm{E}\left[\mathrm{Y}_{\mathrm{i}}\right]\right)+\mu_{\mathrm{X}_{\mathrm{a}}}^{\prime 2}\left(\mathrm{E}\left[\mathrm{Y}_{\mathrm{i}}\right]\right) \operatorname{Var}\left[\mathrm{Y}_{\mathrm{i}}\right]\right\}
\end{aligned}
$$

Taking reliability index $\beta$ into consideration of path choices, the more the $\beta$ is, the more the reliability is. In this case, although routing distance of the path might not be the shortest, but the probability of arriving destination in time using this route is higher.

Using the real street network in Taipei city center as example, as shown in Figure 4 and choosing a pair of $O D$, a comparison of shortest path and more reliable path is conducted. It is assumed with different departure times, which are AM 7:00, AM 8:00 and AM 9:00. The shortest expectation travel time and the most reliability path result from the algorithm is shown in Table 1. The expectative travel time is the shortest path travel time plus 10 minutes, then using the definition of $\beta$ under the expected arrival time is the minimal travel time plus $10 \mathrm{~min}$, we could calculate the probabilities of in time arrival within the expected travel time of the shortest path and most reliable paths from the same origin node to arrive destination node with different departure times.

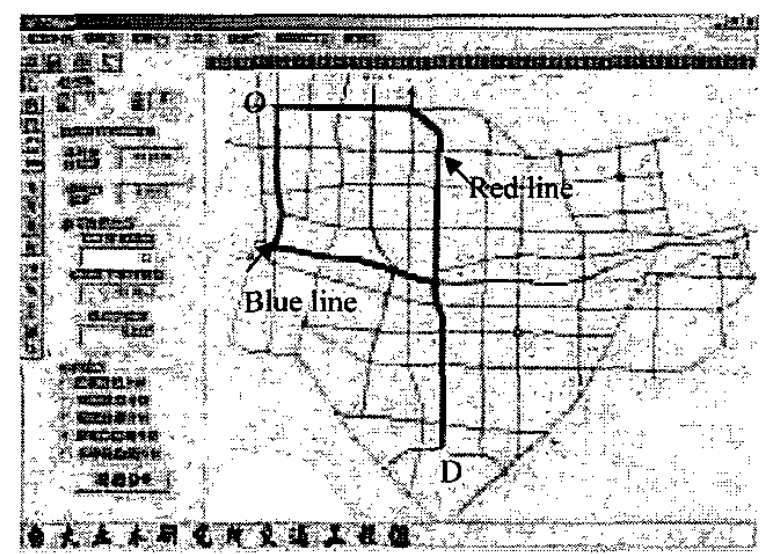

Note: blue line is the more reliable path; red line is the shortest path of travel time, starting at 8 o'clock, based on the travel time of Fu and Rilett's formula

Figure 4. The example street network in Taipei

The change ratio of probability is higher than the increased ratio of travel time. It shows might it be worth to take a longer travel time but get more reliability.

Table 1: Comparison with different departure time

\begin{tabular}{|l|c|c|c|c|c|c|}
\hline & \multicolumn{2}{|c|}{ AM 7:00 } & \multicolumn{2}{c|}{ AM 8:00 } & \multicolumn{2}{c|}{ AM 9:00 } \\
\cline { 2 - 7 } & $\begin{array}{c}\text { Travel } \\
\text { time }\end{array}$ & $\begin{array}{c}\text { Prob. } \\
\text { of } \\
\text { arrive } \\
\text { in } \\
\text { time }\end{array}$ & $\begin{array}{c}\text { Travel } \\
\text { time }\end{array}$ & $\begin{array}{c}\text { Prob. } \\
\text { of } \\
\text { arrive } \\
\text { in time }\end{array}$ & $\begin{array}{c}\text { Travel } \\
\text { time }\end{array}$ & $\begin{array}{c}\text { Prob. of } \\
\text { arrive } \\
\text { in time }\end{array}$ \\
\hline $\begin{array}{l}\text { Shortest } \\
\text { path }\end{array}$ & $\mathbf{5 4 . 6 6}$ & $\mathbf{0 . 7 3}$ & $\mathbf{8 4 . 8 7}$ & $\mathbf{0 . 7 3}$ & $\mathbf{6 1 . 7 2}$ & $\mathbf{0 . 8 0}$ \\
\hline $\begin{array}{l}\text { Reliabili } \\
\text { ty Path }\end{array}$ & $\mathbf{5 4 . 6 6}$ & $\mathbf{0 . 7 3}$ & $\mathbf{9 3 . 3 8}$ & $\mathbf{0 . 8 5}$ & $\mathbf{6 6 . 8 8}$ & $\mathbf{0 . 9 8}$ \\
\hline $\begin{array}{l}\text { Diff. } \\
\text { (\%) }\end{array}$ & & & $\mathbf{1 0 . 0}$ & $\mathbf{1 6 . 4}$ & $\mathbf{8 . 3}$ & $\mathbf{2 2 . 5}$ \\
\hline
\end{tabular}

\subsection{Using multi-distribution formula}

Dividing AM 7:00 to 9:00 to several time intervals and setting the travel time distribution of the link when 
arriving in the link within each time interval, a time dependent travel time is then established. The travel time distribution is assumed as normal distribution.

Taking the same OD pairs above and the same departure time, two paths are obtained. One is minimum travel time of shortest path and the other is the more reliable path with higher probability of arriving destination in time

The result represents as Table 2. Assume the expected travel time is the shortest path travel time plus 10 minutes, then basing on the definition of $\beta$, the probabilities for arriving the destination in time are obtained of the shortest path and most reliable path from the same origin node to arrive destination node in time with different departure time.

According to Table 2, we find that the difference percentage of probability of arriving in time of the more reliable path is less than the ratio of travel time increased in comparison with the shortest path. Furthermore, the path using Fu and Rilett's method, s. Figure 4 is different from the path using multi-distribution Method, s. Figure 5.

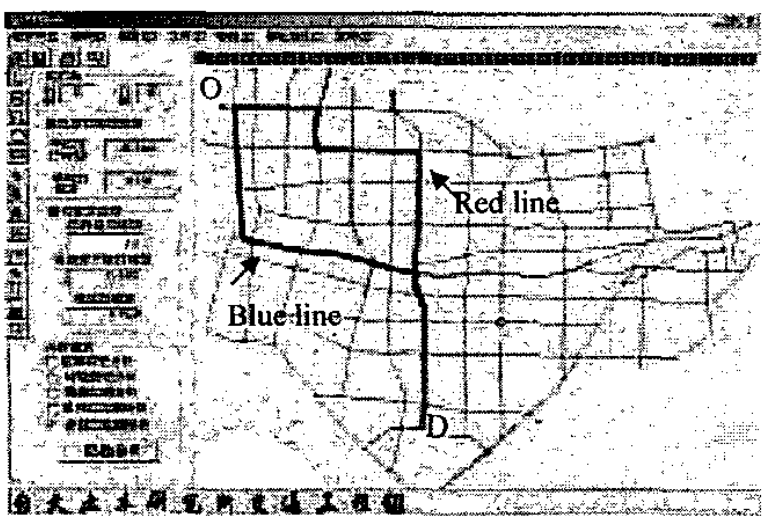

Note: Blue line is the more reliable path; red line is the shortest path, started at 8 o'clock, based on the multi-distribution travel time formuła

Figure 5 Example street network in Taipei

Table 2: Comparison with different departure time

\begin{tabular}{|l|c|c|c|c|c|c|}
\hline & \multicolumn{2}{|c|}{ AM 7:00 } & \multicolumn{2}{c|}{ AM 8:00 } & \multicolumn{2}{c|}{ AM 9:00 } \\
\cline { 2 - 7 } & $\begin{array}{c}\text { Travel } \\
\text { time }\end{array}$ & $\begin{array}{c}\text { Prob. of } \\
\text { arriving } \\
\text { in time }\end{array}$ & $\begin{array}{c}\text { Travel } \\
\text { time }\end{array}$ & $\begin{array}{c}\text { Prob. of } \\
\text { arriving } \\
\text { in time }\end{array}$ & $\begin{array}{c}\text { Travel } \\
\text { time }\end{array}$ & $\begin{array}{c}\text { Prob. of } \\
\text { arriving } \\
\text { in time }\end{array}$ \\
\hline $\begin{array}{l}\text { Shortest } \\
\text { path }\end{array}$ & $\mathbf{4 2 . 1 7}$ & $\mathbf{0 . 7 2}$ & $\mathbf{4 8 . 3 4}$ & $\mathbf{0 . 7 2}$ & $\mathbf{3 6 . 8}$ & $\mathbf{0 . 7 4}$ \\
\hline $\begin{array}{l}\text { Reliable } \\
\text { Path }\end{array}$ & $\mathbf{4 7 . 9 2}$ & $\mathbf{0 . 7 4}$ & $\mathbf{5 3 . 9 1}$ & $\mathbf{0 . 7 9}$ & $\mathbf{4 0 . 0}$ & $\mathbf{0 . 7 5}$ \\
\hline $\begin{array}{l}\text { Diff. } \\
\text { (\%) }\end{array}$ & $\mathbf{1 3 . 6}$ & $\mathbf{2 . 7}$ & $\mathbf{1 1 . 5}$ & $\mathbf{9 . 7}$ & $\mathbf{8 . 7}$ & $\mathbf{1 . 3}$ \\
\hline
\end{tabular}

\section{Conclusion}

This paper discussed the problem of a stochastic network and created a new method to conduct dynamic stochastic time varying networks shortest paths problem by reliability index. Under the consideration of reliability, although the more reliable path will have longer routing distance, but its probability of arriving destination in time is higher than the shortest path. For rescue work post great earthquake, to ensure the vehicle to arrive at the disaster area in time is also important. This result could be applied in ATIS of ITS, offering the shortest travel time and the most reliable path. However, how to get the real data of the travel time distribution and time dependent travel time function is the first issue to apply this method. In this paper, the double labels algorithm can be applied to generate the more reliable path very conveniently. However, the path could not be the most reliable path of the whole network. The path is the most reliable path linked with the shortest path and not too long travel time than the shortest path. This method can obtain the shortest path and the most reliability path at the same time more efficiency than using $K$ shortest path algorithm. Following this concept developed in this paper, the shortest problem will be extended to discuss the most reliable path in the future.

\section{References}

[1] A.. H-S. Ang, and W. H. Tang, "Probability Concepts in Engineering Planning and Design", Volume II :Decision, Risk, and Reliability, Rainbow-Bridge, 1984

[2] L. Fu, and L.R. Rilett; L.R., "Expected Shortest Paths in Dynamic and stochastic Traffic Networks", Transportation. Res.-B, Vol. 32, No. 7, PP. 499-516, 1998

[3] Hall, and W. Randolph, "The fastest Path through a Network with Random Time-dependent Travel Times", Transportation Science, Vol.20, No. 3, pp.182-188, 1986.

[4] M. Hooks, D. Elise, and H. S. Mahmassani,. "Least Possible Time Paths in Stochastic Time-Varying Networks", Computers Ops. Res. Vol. 25, No. 12, pp. 1107-1125, 1998

[5] M. Hooks, D. Elise, and H. S. Mahmassani, "Least Expected time Paths in Stochastic Time-Varying Transportation Networks", Transportation Science, Vol. 34, No. 2, pp. 198-215, 2000.

[6] Ziliaskopoulos, K Athansios, and H. S. Mahmassani, "Time-Dependend Shortest-Path Algorithm for Real-Time Intelligent Vehicle Highway System Applications", TRR 1408, pp. 94-100, 2000. 